PERSONAL VIEW - THE LARGE SCALE STRUCTURE OF THE UNIVERSE

\author{
M. S. Longair \\ Mullard Radio Astronomy Observatory \\ Cavendish Laboratory, Cambridge
}

I should explain at once that I very much regret that it is the Chairman of the Scientific Organising Committee who has been pressganged by his Committee into giving the concluding remarks at this symposium. I obviously cannot take an unbiassed view of the proceedings. I have therefore refrained from calling this a "survey" or "concluding remarks" - it is a personal view of those aspects of this symposium which have struck me as particularly interesting - I will make no attempt to provide a complete survey of the last five days.

It is the privilege of those landed with the task of attempting to bring a symposium to a rousing conclusion to offer comments which may be termed "philosophical", "sociological" or "banal", depending on your point of view, and I will not be the exception to this rule. First of a11, I often think conferences are not particularly successful at communicating information or ideas, in the sense of what people really think about different problems. Partly this is because the symposium format does not allow enough time for proper discussion of particularly thorny points, partly it is because people are too modest in expressing their worries (or perhaps their ignorance) and partly it is because many people who have serious criticisms of a particular piece of work do not express their views, perhaps because they think the work under discussion is obviously wrong, perhaps because they cannot be bothered or perhaps because they have said too much already. I don't know how to overcome these problems. What I will try to do is to expose some of the questions and worries which were running through my mind during the symposium.

A second point which struck me is that there are basically two types of astrophysicist - those who like simple things and those who love complicated things. This applies to both observers and theoreticians. This dichotomy is particularly apparent in a subject like the large-scale structure of the Universe. We are all aware of the tremendous complexity of the Universe and sooner or later we will have to account for every little bit of it. However, this should not prevent us seeking the overall regularities in the Universe. What many of us 451

M. S. Longair and J. Einasto (eds.), The Large Scale Structure of the Universe, 451-461. All Rights Reserved. Copyright $\odot 1978$ by the IAU. 
want to find out are the basic principles according to which the large scale structures form and evolve and by which the complexities which we observe today came about. You will gather from this remark that I am a proponent of the "simplistic" school of astrophysics.

Finally, before tackling the science, it is important to remember the methodology involved in studies of the large scale structure of the Universe. It is not simply a question of observations and theory but rather a complex interaction of observations, their interpretation and theory. Theories which are too strongly dependent on interpretation of observations must live dangerously. The oftener the theories confront the observations directly, the more secure the validation or otherwise of the theory.

The Scientific Organising Committee had wel1-defined objectives in designing the programme. In the first three parts, we considered large scale structures in the Universe in order of increasing dimension and discussed the astrophysical problems which they pose in the context of the observations:

1. GALAXIES IN SMALL GROUPS.

2. CLUSTERS OF GALAXIES.

3. LARGE SCALE SYSTEMS.

Natura11y, these discussions involved consideration of the evolution of these systems over cosmological time-scales but, as we heard, there is little direct observational evidence for evolution in these systems. Therefore, part 4 was devoted to related fields in which there may be direct evidence for the effects of cosmological evolution

4. OBSERVATIONAL EVIDENCE FOR COSMOLOGICAL EVOLUTION.

Having then completed the description of the large scale properties of the Universe as we know it, we then grasped the thorny problem of the origin of these structures

5. THE FORMATION OF STRUCTURE IN THE UNIVERSE.

I will devote most attention to optical observations of the large scale structure of the Universe in Sections 1,2 and 3 before considering the theory of such systems in Section 4. In the remaining sections, I will consider evidence for cosmological evolution and the origin of large scale systems.

\section{GALAXIES IN SMALL GROUPS}

De Vaucouleurs' classical work on groups of galaxies in the Reference Catalogue of Bright Galaxies was referred to many times. The problems of establishing group membership and their reality as bound self-gravitating systems came through clearly as the most serious problems, the resolution of which has repercussions throughout many different aspects of cosmology, for example, the mean density of matter 
in the Universe and the nature of the hidden mass which must be binding relaxed groups and clusters.

Despite the fact that many different workers were using basically the same material, there were two distinct schools of thought about what the mean mass to luminosity ratio, M/L, of groups of galaxies within about $20 \mathrm{Mpc}$ really is. One school believes $\langle\mathrm{M} / \mathrm{L}\rangle \approx 5-10$, the other $\langle M / L\rangle \sim 100$. First of all, it should be emphasised that this is only a factor of 10 which is not too bad by some astronomical standards but most people feel the discrepancy is too big. Fortunately, the methodology used by each observer was described clearly and from this we (or rather they) should be able to decide whether there is a real discrepancy or not. Many times we heard of the importance of including properly the high velocity members of the group since they make a large contribution to its kinetic energy and hence require large masses to bind them to the group. I very much hope this symposium will help bring together these workers so that they can decide if there is a real discrepancy and whether or not it is entirely a question of group membership.

It did strike me that there is no very good reason why a11 groups should have the same $\mathrm{M} / \mathrm{L}$ ratio and partly the discrepancy might be due to the selection of different types of groups by different observers. It was also not clear whether or not the discrepancy, if real, can be reconciled within a single picture. In view of the uncertainty about what form the binding mass of groups and clusters takes, I would be most surprised if one could not reconcile the different values within a single picture.

Having raised the question of the form of the binding mass of clusters and groups, I was somewhat surprised that we heard relatively little about its nature. Is this because everyone agrees it must be there but they have given up for the present trying to choose among the various possibilities? Hegyi presented evidence for an almost spherical halo around NGC 4565 with radius $\sim 40 \mathrm{kpc}$ and Lynden-Be11, in his talk in which within 25 minutes he changed the Hubble constant and the size and mass of the Galaxy, suggested that there may be a massive halo around our Galaxy. Against this view, Karachentsev showed that the M/L ratio for widely separated double galaxies is "normal", i.e. 8-10. This last approach would appear to be a most promising method for obtaining further real data about massive haloes. Another manifestation of massive haloes may be Einasto's hypergalaxies. The velocities of satellite galaxies in hypergalaxies must give dynamical information about the total mass of these systems and it is to be hoped the objects in his first catalogue of hypergalaxies will be the subject of intensive study.

I was particularly impressed by the vast amount of high quality redshift data which is now becoming available for nearby groups, both from optical studies and from HI velocity data. I feel that so much 
data is now being accumulated that many of the problems of the nearby groups must soon be clarified.

\section{CLUSTERS OF GALAXIES}

The optical properties of the great clusters received relatively little attention, perhaps because many of their properties are now well estbalished. One point which strikes me on reading the literature on the great clusters is that there are many classification schemes but all of them seem to boil down to one continuous classification in which there are three basic types, Regular, Intermediate and Irregular clusters. This point is made very clearly in Neta Bahcall's review of clusters of galaxies to appear in Annual Reviews of Astronomy and Astrophysics 1977. An abbreviated version of her table is given in Table 1 for reference. I believe that most of the interesting astrophysical aspects of clusters are contained within this simple scheme.

Table 1

Classification of clusters of galaxies (after N. Bahca11)

\begin{tabular}{|c|c|c|c|}
\hline $\begin{array}{l}\text { Classification } \\
\text { or Property }\end{array}$ & REGULAR & INTERMEDIATE & IRREGULAR \\
\hline Zwicky & Compact & Medium-Compact & Open \\
\hline Bautz-Morgan & I, I-II, II & $(I I), I I-I I I$ & $(I I-I I I), I I I$ \\
\hline Rood-Sastry & $c D, B,(L, C)$ & $(L),(F),(C)$ & $(F), I$ \\
\hline Content & E11iptical rich & Spiral poor & Spiral rich \\
\hline $\mathrm{E}: \mathrm{SO}: \mathrm{S}$ ratio & $3: 4: 2$ & $1: 4: 2$ & $1: 2: 3$ \\
\hline Symmetry & Spherical & Intermediate & Irregular Shape \\
\hline $\begin{array}{l}\text { Central } \\
\quad \text { Concentration }\end{array}$ & High & Moderate & Very 1 ittle \\
\hline Central Profile & Steep gradient & Intermediate & Flat gradient \\
\hline Mass segregation & Marginal & Marginal & No segregation \\
\hline
\end{tabular}

\section{LARGE SCALE SYSTEMS}

To me, some of the most exciting results presented at this symposium concerned the structure of the Universe on the largest scales. Everyone seemed to agree about the existence of superclusters - de Vaucouleurs' description of the local supercluster, Tully's film of local supercluster and Peeble's $10^{\prime} \mathrm{x} 10^{\prime}$ plot of the Shane-Wirtanen counts are convincing direct evidence of systems on scales $\sim 30-100 \mathrm{Mpc}$. 
But perhaps even more surprising are the great holes in the Universe. Peeble's picture, Einasto's analysis of the velocity distribution of galaxies which suggests a "cell-structure" and Tifft's similar analysis argue that galaxies are found in interlocking chains over scales $\sim 50-$ $100 \mathrm{Mpc}$ forming a pattern similar to a lace-tablecloth. The holes are particularly interesting since they might appear to be at variance with the idea of continuous clustering on all scales which we have been educated into understanding from the original work of Kiang up to the more recent analyses of Peebles and Abe11. I do not believe there is any basic contradiction here - one cannot expect a covariance function approach to reproduce sharp features like holes. I am still a firm believer in the basic correctness of the results of the covariance analysis - more detailed astrophysical arguments will have to account for details such as holes which are about $10 \mathrm{Mpc}$ in size and void of bright galaxies.

On the very largest angular scales, patchy galactic obscuration becomes a problem but we heard from Kalinkov that there remains structure on these scales, "third-order clustering", when the analysis is restricted to high galactic latitudes. His beautiful pictures in which the distribution of clusters was convolved to a very large angular scale suggested two main groupings of clusters. If these structures are real, they must be the largest associations of optical galaxies known in the Universe. One wonders whether their existence is consistent with the isotropy of the distribution of extragalactic radio sources and of the microwave background radiation.

\section{THEORETICAL STUDIES}

Turning now to theoretical studies of these structures, I must begin with Peebles' covariance function. I have always regarded this as one of the most beautiful pieces of analysis of the distribution of objects in the Universe and, although the procedure is fairly complex, the result is amazingly simple. The covariance function on all scales from $\sim 30 \mathrm{kpc}$ to $\sim 30 \mathrm{Mpc}$ has a simple power-1aw form $\xi(\mathrm{r}) \propto \mathrm{r}^{-1.77}$. Within this picture one can account for structures observed on different scales - de Vaucouleurs' groups and local supercluster, Zwicky's superclusters, Abell's associations of Abe11 clusters, etc. There are of course further complications which result from more detailed analyses (such as chains of galaxies and clusters and the "holes" in the Universe), but it would seem that any satisfactory theory of the origin of clustering in the Universe should account for this.

This is what is so impressive about the work of Aarseth and his colleagues in their simultations of galaxy clustering. The model is of great basic simplicity. Galaxies are regarded as "fuzzy particles" and the particles interact only through their mutual gravitational attraction. The amazing result of these computations is a more-or-less perfect power-law with slope close to that found by Peebles which 
extends over 4 orders of magnitude in physical scale. Granted the simplicity of the basic model, I find it staggering that the end point of these calculations should end up giving so close agreement with the observed covariance function for galaxies in the real Universe. As I understand the results of this work, the predicted power-law covariance function is insensitive to many of the initial assumptions. It should also be noted that this power-law results from a myriad of non-1inear interactions between particles and that there is as yet no simple way of deriving this result by physical arguments.

Peebles mentioned some of his worries about the way in which the problem was set up, in particular that on small enough scales the density fluctuations must have $\delta \rho / \rho \geqslant 1$ and hence non-1inear effects are already important. I would tend to argue the other way round. One of the great strengths of the approach of Aarseth and his colleagues is that they set about their simulations using sufficient particles (4000) to mimic our region of the actual Universe at the present day. Thus, if the general picture of collapse is correct, they are mimicking exactly what must have happened to the actual Universe and it too must have had to worry about the fact that the fluctuations were already large on a small enough scale. I interpret the success of the computations as telling us something about the initial conditions from which large scale structures evolved.

Having been elated by these results, which, incidentally, I classify as "simple", I then went through my low point at the conference when de Vaucouleurs said in discussion that these simulations looked nothing like the real Universe!! I have been privately canvassing opinions about this since then and most people seem to think the simulations do not look too unlike the real Universe. It is true that we do not see al1 the complexity of the real Universe but I feel we at least have the beginnings of the picture. We do not perhaps see the well developed "cel1-structure" in these simulations (although I believe I can see things like them) but it must be remembered that the computations were only made with 4000 "soft" point masses. One can envisage in addition many astrophysical processes which would change the details of the simulations and perhaps make them look more like the real Universe but which would not change the overall result of the present simulations. I interpret de Vaucouleurs' remark as encouragement for us all to work harder!!

Ostriker's presentation of the dynamical evolution of clusters using only Newtonian gravity was, to me, wholly convincing and need not be amplifed here. The scheme can account naturally for the various types of cluster listed in Table 1 as well as phenomena such as the formation of $\mathrm{cD}$ galaxies at the centres of clusters, possibly with multiple nuclei. In addition, we can all see reasonable ways in which hypergalaxies and even Vorontsov-Velyaminov's "nests" of galaxies could come about. 
The one thing which I found missing in this presentation was the problem of the $\mathrm{M} / \mathrm{L}$ ratio for clusters. Ostriker made the convincing remark that regular clusters, such as the Coma cluster in which the velocity distribution is Maxwellian, is just as much in equilibrium as the inside of a star and hence there is no question of the mass being missing - it is merely not visible. We heard the important result that all the hidden mass could not be in the galaxies because dynamical friction would then be so important that the clusters would today be in a state of extreme "stripping" and "cannibalism" which apparently has not yet happened. One guesses that the binding mass is some form of "stellar" distribution which follows roughly the galaxy distribution maybe it is in the form of very faint stars, possibly neutron starts or blackholes. One wonders exactly what the nature of the diffuse light in the Coma cluster is which was described by Melnick and his colleagues. A conservative intuition suggests that the binding mass may be no more than low-mass stars which are easily stripped from galaxies and now belong to the cluster as a whole.

I have omitted so far all mention of the radio and X-ray properties of clusters. This is because at the present day I feel they are perhaps diagnostic tools for studying more detailed aspects of cluster evolution rather than the basic problem of cluster formation. However, I must immediately emphasise their central importance in the study of the astrophysics of the evolution of gas in clusters and of extragalactic radio sources. In addition, studies of extragalactic radio sources provide a powerful method of detecting distant rich clusters of galaxies. The X-ray satellite HEAO-B will be able to observe X-ray cluster sources at cosmological distances and these will be of central importance in studying the early evolution of clusters. The reviews by Perola and Ekers and by Culhane of radio and X-ray observations of clusters respectively indicate clearly the wide range of exciting astrophysics which these disciplines have contributed.

At this point in the symposium, we discussed explicitly the question of the mean density of matter in the Universe, a problem which had been casting its shadow over a number of the discussions. The present situation is admirably summarised in the discussion chaired by Tammann. The range of mass-to-light ratios considered ranges from about 5 to 200 and the corresponding values of the density parameter $\Omega$ from about 0.02 to 0.3. There were a few suggestions of higher values, $\Omega \sim 0.5-0.8$. What was particularly interesting about the discussion are the "new" methods of estimating $\Omega$ - from perturbations of the Hubble flow, from the "statistical virial theorem", from the correlation of the peculiar motions of galaxies with density perturbations. A11 of these methods require large bodies of high quality redshift data but one should be reasonably optimistic that these observations will become available in the foreseeable future. The consensus view appeared to be that values of $\Omega \simeq 0.1$ are favoured by $I$ do not think anyone would want to exclude values of $\Omega \sim 1$ at present. 


\section{EVIDENCE OF COSMOLOGICAL EVOLUTION}

None of the presentations in Section 4 of the programme actually provided observational evidence for the evolution of large scale systems but rather evidence for the evolution of very rare, super-luminous objects - quasars and radio galaxies. The $\mathrm{V} / \mathrm{V}_{\max }$ test for quasars and the counts of radio sources are still by far the most significant pieces of evidence for the cosmological evolution of any class of object in the Universe. Broadly interpreted, they suggest that the Universe went through a period of violent activity when it was about 0.1-0.2 of its present age. Quasars and radio galaxies were much more common events at these epochs than they are now by a factor of about $10^{3}$. It is my belief that we will eventually learn a great deal about when galaxies and clusters formed and also about the evolution of the environments of radio sources from these studies as $I$ outlined in my lecture.

In comparison, the evidence for the evolution of galaxies and clusters is sparse. Tinsley revieved the few available pieces of evidence and the problems of interpretation. However, one can confidently expect the amount of data on these topics to increase dramatically over the next few years as much more data is accumulated by the increasing number of optical telescopes in the 4-6 m class. In the mid 1980's we have the prospect of the NASA Space Telescope which everyone believes will revolutionise extragalactic studies just as the construction of the Hale 200-inch telescope opened up a new era of extragalactic studies in the 1940 's.

Two topics struck me as being ripe for immediate attention. First, Karachentsev and Kopylov presented counts of galaxies to 24 th magnitude made with the SAO 6-m telescope which appeared to be in remarkable agreement with the predictions of uniform world models. Quite different results were reported in discussion by Abe11, de Vaucouleurs and Tinsley. The problem of making self consistent counts of galaxies to faint magnitudes are well known, in particular the problem of knowing the magnitude scale at these faint magnitudes. Tinsley has emphasised how sensitive the counts of galaxies are to evolutionary changes with cosmological epoch. An interesting comparison is with the counts of radio sources where strong evolutionary changes are observed. The problem in interpreting the radio source counts is that the theory of the origin and evolution of individual radio sources is at too primitive a stage to provide astrophysical foundations for the interpretation of this phenomenon. On the other hand, the interpretation of the optical spectra of galaxies is much more secure and thus may provide more definite evidence on the evolutionary history of galaxies as a whole.

The second point relates to the first - namely, young galaxies, if they formed at redshifts $\mathrm{Z} \sim 5-10$, may well be detectable as nonvariable stellar objects similar to quasars. Sunyaev expressed this view (which I share) in discussion. It is particularly intriguing to speculate what the nature of Bolton's non-variable radio-quiet quasars 
is. These are exactly the types of object which Sunyaev and I believe may be strong candidates for young galaxies with redshifts $Z \sim 3-5$. The discovery of such objects would put the whole subject of the formation and early evolution of galaxies on a firm observational foundation and hence programmes to search for them are well worth an intensive effort.

\section{THE ORIGIN OF STRUCTURE IN THE UNIVERSE}

Finally, we come to the problems of the origin of structure in the Universe, probably the most fundamental question of all. I and many of my colleagues have been deeply impressed by the work of the Moscow groups in this field. I regard their work as among the most exciting and imaginative in modern astrophysics and their efforts have made major contributions to our understanding of these basic problems. However, as we have heard, widely differing theories can account for the observed structure of the Universe - specifically, these models are the adiabatic and isothermal models developed by Zeldovich and his colleagues and the whirl theory described by Ozernoi. The basic problem is that the end product of the models is determined to a large extent by the initial conditions. In all models, there is no convincing physical explanation of the initial spectrum of perturbations which eventually leads to the formation of galaxies, clusters, superclusters, etc. Zeldovich expressed the belief that eventually we will be able to understand this once we have a better understanding of the physics of the very earliest stages, including the quantum epochs, of the evolution of the Universe. This is an exciting prospect but I wonder how many astrophysicists believe this is really attainable in the foreseeable future.

According to Zeldovich and Ozernoi all three theories mentioned above can account for observed structures in the Universe. I confess that I was disappointed not to hear critiques of these models by the proponents of the different theories. Many of us are aware of the hot disputes between the Moscow schools which have stimulated splendid pieces of astrophysics. Personally, I would have liked to hear more of the physical problems of these theories rather than of their successes. This is not just a question of theoretical interest but one of immediate importance for observation which I will come to below.

A11 of us must have been impressed by the film of the development of "pancakes" by Doroshkevich and his colleagues and by the remarkable resemblance to the cel1-structure of the Universe described by Einasto, Tifft and others. Intuitively, I am attracted to the adiabatic picture of the origin of galaxies, principally because of the simplificity of the picture. There is a marked contrast in complexity between this adiabatic picture and the whirl picture. To oversimplify, in the former, the non-linear stages of collapse happen once when large scale structures collapse at $\mathrm{Z} \sim 5-10$ whereas in the latter the theory is nonlinear at all epochs. In the adiabatic model, the Universe may be considered to be effectively isotropic and homogeneous up to the epoch 
$\delta \rho / \rho \sim 1$ and hence al1 the attractions of the canonical hot big-bang model are also valid in this model. This is not necessarily so in the whirl model. The development of the turbulent spectrum in the radiation dominated phase is the result of non-linear interactions and galaxy formation results from the collapse of eddies which have a supersonic turbulent spectrum after recombination. As mentioned by Jones, the theory is constantly fighting the problem of the dissipation of turbulence which must be continuously regenerated by the (non-linear) decay of larger-scale eddies coming through the horizon. The very early stages of evolution of this model are also much more complex than in the adiabatic model and it is not clear that it is consistent with the isotropy of the microwave background radiation and with cosmological synthesis of the light elements. However, just because the whir1 theory is more complicated, it does not mean that it is wrong and $I$ heard no piece of evidence at the symposium which showed that the whirl theory was in immediate danger of suffering a mortal blow.

The problem with all these theories is one of observational validation. The methodological problem is that all models are designed to produce the observed Universe at the present epoch. There are very few observational tools by which the early development of these models can be tested, the only viable one at present being observations of the microwave background radiation. The most promising test is the observation of fluctuations in the intensity of the microwave background radiation which, according to most workers, gives us information about the amplitudes and velocities associated with density perturbations at or immediately after the epoch of recombination. We heard of the very low limits to intensity fluctuations now being obtained with instruments such as the Ratan-600 radio telescope by Parijskij, $\Delta \mathrm{T} / \mathrm{T} \leqslant 10^{-4}$ or better. The problem as I see it is that, although all theories are still consistent with these upper limits, as described by Sunyaev and by Ozernoi, it is not at all clear how the theories could be distinguished even if fluctuations are observed. In addition, as I have emphasised, there is no independent method of obtaining information about these early epochs. In this situation we are particularly in need of clarification of the theoretical difficulties of the models so that we can judge for ourselves how we are to interpret the data.

A final important point about these observations is the following. The sensitivities and angular scales which are most important for studying these problems are such as to require either very large amounts of observing time on existing radio telescopes or the construction of space experiments with total cost of $\sim 50-100 \times 10^{6} \$$. The enthusiasm of radio astronomers (and more important, the grant-awarding agencies) is considerably dampened if they learn that following the experiment we will have got no further forward in the resolution of which theory is the best description of how the Universe has evolved. Sunyaev described very clearly the many possible sources of fluctuations in the microwave background radiation which are all expected to become important at about the same intensity level as those due to collapsing proto- 
structures in the Universe $\Delta \mathrm{T} / \mathrm{T} \sim 10^{-5}$. Everyone would agree that the measurement of fluctuations in the microwave background radiation would tell us something important about the Universe but I'm not sure that we will be able to agree what that is.

These remarks are not intended to discourage theorists or observers. They are intended to spur theorists to great efforts to eliminate at least some of the many possible theories of the origin of fluctuations in the microwave background radiation and possibly of rival theories of galaxy and cluster formation.

I hope you have enjoyed the symposium as much as I have. On behalf of the Scientific Organising Committee, I thank you all for coming and making it such a memorable occasion. 